\title{
GIEMSA BANDING AND CHIASMA DISTRIBUTION IN THE DESERT LOCUST
}

\author{
D. P. FOX and K. C. CARTER \\ Department of Genetics, University of Aberdeen, 2 Tillydrone Avenue, Aberdeen AB9 2TN \\ G. M. HEWITT \\ School of Biological Sciences, University of East Anglia, University Plain, Norwich, NOR $88 C$
}

Received 3.v.73

\section{Summary}

Techniques which produce C-bands and G-bands in man also produce bands in the chromosomes of the Desert locust. These bands are not confined to the regions of constitutive heterochromatin.

The chromosome regions which stain with these techniques form few or no chiasmata during male meiosis.

Other factors are implicated in the control of chiasma frequency in the unbanded chromosome regions.

\section{INTRODUGTION}

A LARGE number of techniques is now available for producing differential staining within the chromosomes of the human karyotype. The resulting chromosome bands have been designated $\mathrm{C}$ (constitutive heterochromatin), $G$ (Giemsa), $Q$ (quinacrine) and $R$ (reverse-Giemsa) bands by the Paris Conference (see Cytogenetics, 11, 313-360 (1972)). Each type of band is produced by different techniques.

Although the exact basis of these staining reactions is at present unclear, it seems likely that for the G-bands at least some component of the chromosomal proteins is involved since the same pattern of G-bands may be obtained with proteolytic enzymes (Seabright, 1971; Wang and Fedoroff, 1972), or detergents (see Lee et al., 1973 and other references contained in that paper) as with the ASG technique (Sumner et al., 1971). Whatever the identity of this chromosomal component, its functional role has remained obscure. In this paper we report that in the Desert locust, Schistocerca gregaria (Forskål), the chromosome regions stained by G-banding techniques have a very low chiasma frequency when compared with adjacent, unbanded regions. It may be that the control of the positions in which chiasmata (and thus crossovers) occur is a major function of these chromosome regions.

\section{Materials and Methods}

The diploid chromosome number of the Desert locust is 23 in the male with the 11 pairs of autosomes and single $\mathrm{X}$ chromosome all having terminal centromeres (John and Naylor, 1961; Fox, 1973). Identification of indi-

Address for correspondence: Dr G. M. Hewitt, School of Biological Sciences, University of East Anglia, University Plain, Norwich, NOR 88C. 


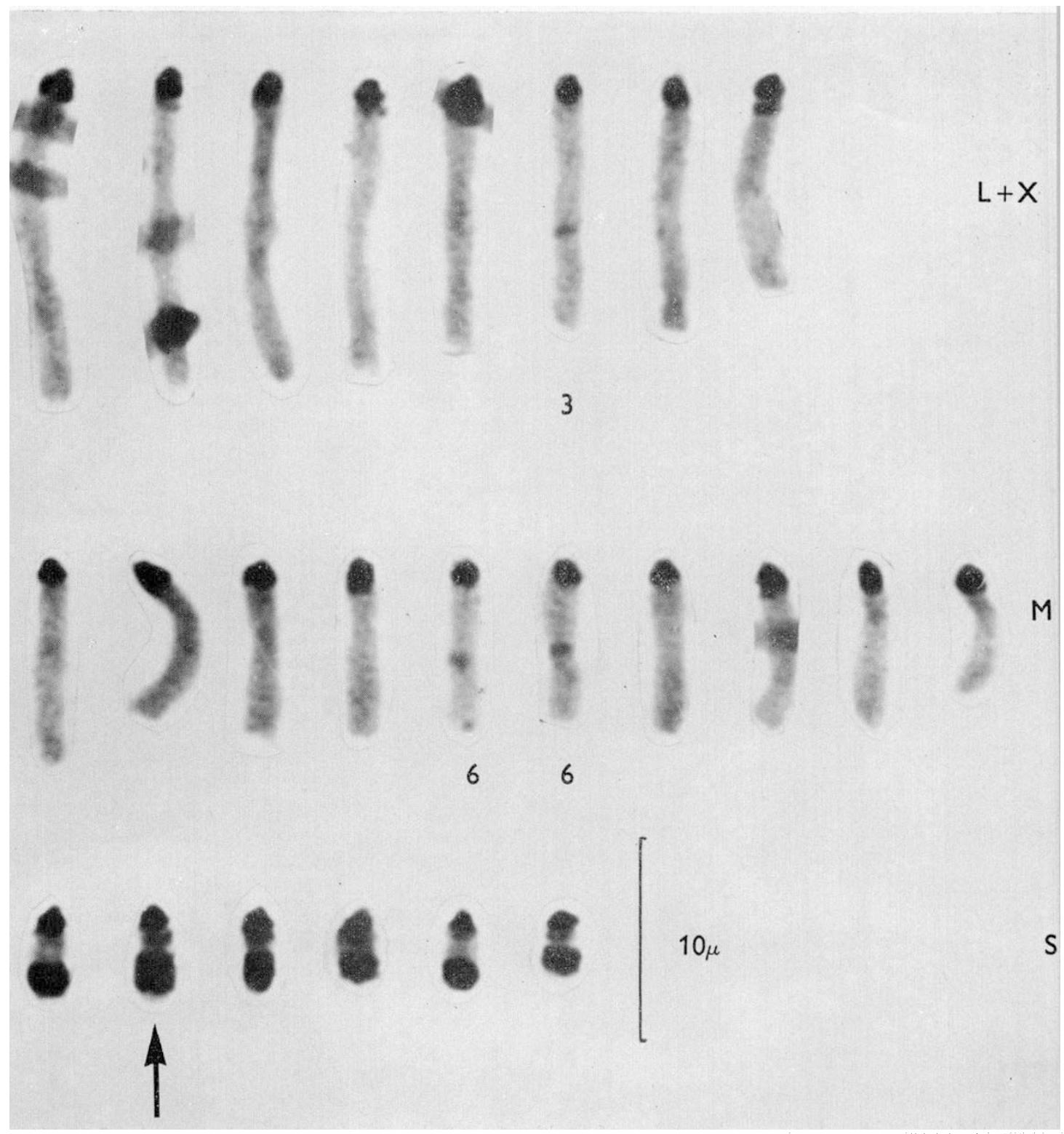

FiG. 1.-G-banding in a G-metaphase neuroblast cell of a female embryo produced with the barium hydroxide technique. One L3 and both M6 homologues can be identified by the $\mathrm{G}-\mathrm{b}$ and at the nucleolus organiser. In some $\mathrm{S}$ chromosomes ( $\uparrow$ ) it is clear that the distal G-band does not extend to the extremity of the telomere. 
vidual chromosomes at diplotene (Fox, 1973) or embryo neuroblast metaphase (Fox, 1966; K. Carter, unpublished work) is complicated by the fact that they differ essentially only in length. However, there is good evidence that, using length as the only criterion, they may be sub-divided into long $(\mathrm{L})$, medium (M) and short (S) groups containing 3,5 and 3 pairs of autosomes respectively. The $\mathrm{X}$ chromosome falls in the L-group in mitotic cells being approximately equal in length to the L3 autosome. In estimating chiasma position the autosomes in each of 541 diplotene cells were ranked in order of decreasing length and each rank position was treated as a homogeneous population. However, in some case several different homologues are known to be present in the population of one rank position (Fox, 1973).

Three techniques have proved to be successful in producing banding in locust chromosomes.

(i) ASG technique (Sumner et al., 1971). Five-day embryos were fixed in alcohol-acetic acid following colcemide treatment $(0.03$ per cent. for 30 minutes) and air-dried slides prepared. The slides were incubated for 1 hour at $60^{\circ} \mathrm{C}$. in $2 \times \mathrm{SSC}(0.3 \mathrm{M} \mathrm{NaCl}, 0.03$ м trisodium citrate). Slides were stained in 1 part Giemsa stock solution to 50 parts phosphate buffer pH 6.8, dried and mounted. Both Michrome and Gurr's R 66 Giemsa proved effective.

(ii) $\mathrm{Ba}(\mathrm{OH})_{2}$ and $2 \times \mathrm{SSC}$ incubation (Sumner et al., 1971). The slides were treated as in (i) above except that they were placed in $0.5 \mathrm{M} \mathrm{Ba}$ $(\mathrm{OH})_{2}$ for 5 minutes prior to incubation in $2 \times \mathrm{SSC}$.

(iii) $4.5 \mathrm{M}$ urea. A modification of the technique of Shiraishi and Yosida (1972) was used. Slides were incubated in $4.5 \mathrm{M}$ urea in phosphate buffer, pH 6.8 at $37^{\circ} \mathrm{C}$. for 10 minutes.

\section{Results AND DISCUSSION}

The ASG and urea techniques characteristically produce G-bands while the $\mathrm{Ba}(\mathrm{OH})_{2}$ technique produces $\mathrm{C}-$ bands. In the locust all these techniques produce the same pattern of bands and these will be referred to as G-bands. The G-band patterns produced in the chromosomes of the Desert locust are relatively simple. In C-metaphases from the neuroblasts of embryos (fig. 1) it can be seen that there is intensive banding at the centric ends of all chromosomes. In some instances (and especially in late prophase cells) it can be seen that three bands are present though with some variation in size and position in different chromosomes. Their relative sizes and positions are indicated in fig. 2.

In the $\mathrm{L}$ and $\mathrm{M}$ group and $\mathrm{X}$ chromosome, G-banding is absent from the rest of the chromosome arm with the exception of a small band in the same region as the nucleolus organiser on chromosomes L3 and M6. These two bands are rather transient and are never seen when the position of the nucleolus organiser is expressed as a secondary constriction. The three smallest autosomes (S9-S11) have a wide G-band at the telomeric end of the chromosome. However, this band does not extend to the extremity of the telomere (fig. 1).

Recently, Fox (1973) has described the detailed pattern of chiasma distribution along the chromosomes of the Desert locust. In fig. 2 along with the pattern of G-banding in each chromosome, the pattern of chiasma frequency variation is shown. Pooled data from 541 diplotene cells of four male $81 / 2-S$ 
individuals were used to derive this frequency pattern. It can be seen quite clearly that there is a negative correlation between the position of G-bands and the frequency of chiasma formation. This correlation is most easily
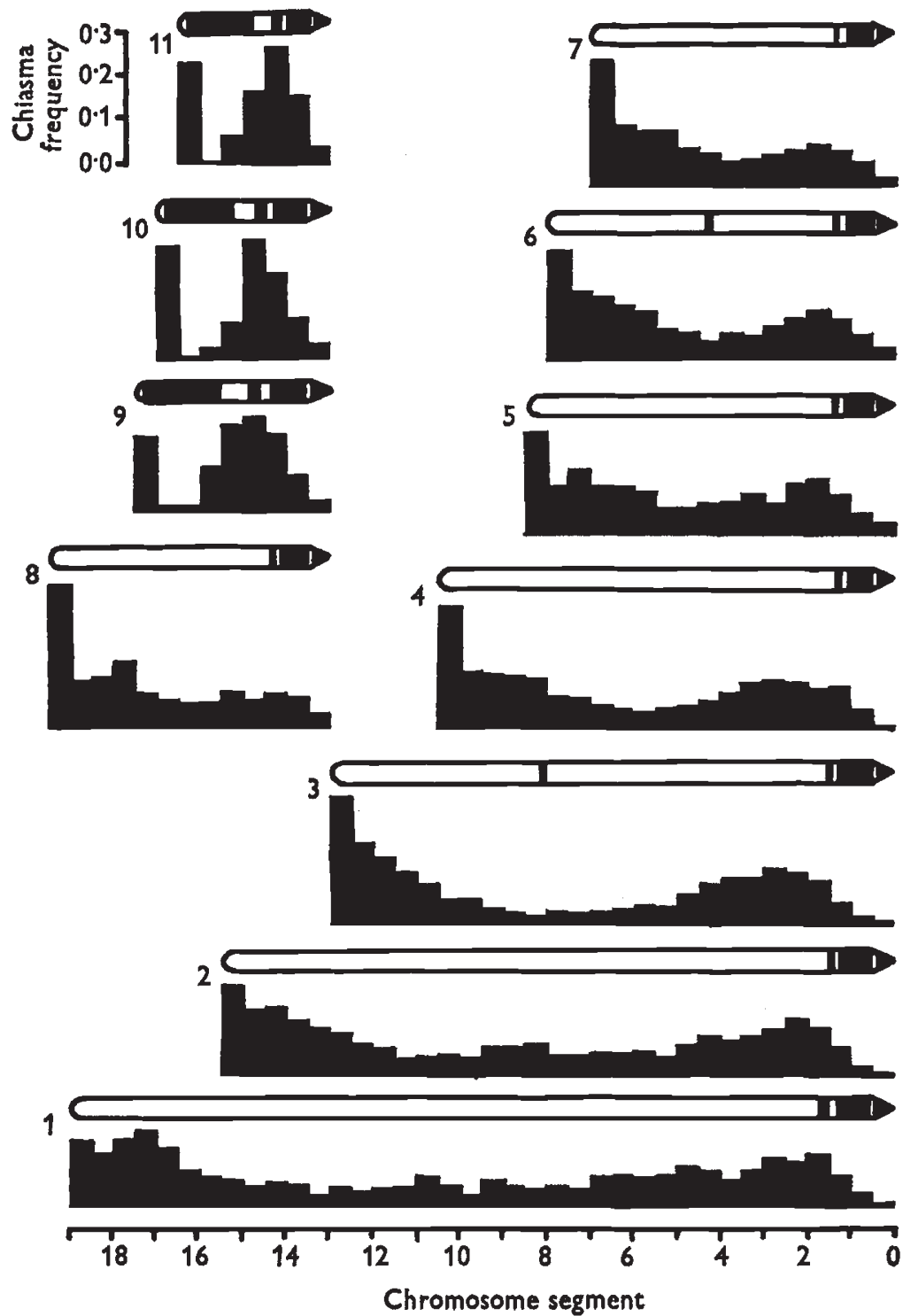

Frg. 2.- The distribution of chiasmata at diplotene (below) and G-bands at mitotic metaphase (above) for the 11 autosome types. The wid th of each histogram bar is 0.5 per cent. of the total autosome length at diplotene and the average chiasma frequency for each bar is given.

seen in the comparison of the short (S9-S11) with the long and medium (Ll-3, M4-8) chromosomes. It is less easy to see an effect of the nucleolus organiser band on chromosomes L3 and M6 but in both these cases the posi- 
tion of the band corresponds with the position of minimum chiasma frequency of these chromosomes (excluding the centric regions). Furthermore, there are other indications of a difference in the pattern of chiasma formation in these two chromosomes since the mean interference distance is greater than in other chromosomes of similar total length (Fox, 1973).

The exact comparison between G-band position in mitotic chromosomes and chiasma position in diplotene chromosomes is complicated by two factors. (i) The determination of chiasma position within chromosomes is subject to some error (Fox, 1973). (ii) While the mitotic chromosomes are more or less uniformly condensed along their length, the diplotene chromosomes are not, being more condensed in the centric regions which are heterochromatic at this stage (Fox, 1973). Thus in comparing chiasma position with mitotic G-band position (fig. 2) we must remember that the centric region is relatively more contracted in the meiotic chromosomes and consequently the centric region with low chiasma formation corresponds well with the intense Giemsa staining. A similar situation seems to hold for the human karyotype in that chromosomes with more G-band material have a lower chiasma frequency than other chromosomes of a similar size which have less G-band material (H. J. Evans, personal communication). We tentatively suggest that this is likely to be a general phenomenon and indeed that this may prove to be a major function of the G-bands in reducing crossing over and thus maintaining supergenes. Preliminary examination (G. M. Hewitt, unpublished work) of Giemsa banding elsewhere in the genus Schistocerca indicates that other species have different patterns of G-bands and a detailed analysis of chiasma distribution in these other species will provide a direct test of this idea. This finding of a negative correlation between G-bands and chiasma position is paralleled by the finding (Savage, 1973; Seabright, 1973) that $\mathrm{X}$-ray induced exchange aberrations are also only formed by the chromosome regions between G-bands.

Two further points deserve emphasis. Firstly, the G-bands of the Desert locust cannot be equated with constitutive heterochromatin for, though the centric regions of all the chromosomes are heterochromatic in that they stain more intensively with orcein (Fox, 1973) and are late labelling in premieotic S-phase (Craig-Cameron, 1971). The other G-banded regions, especially those in the $\mathrm{S}$ chromosomes, are not heterochromatic on these same criteria. Over the years several reports (see John and Lewis, 1965, p. 16) have indicated a possible correlation between heterochromatin and absence of recombination but the coincidence of G-bands with achiasmate regions is both more specific and potentially capable of a molecular explanation. Secondly, the G-bands cannot be the sole mechanism responsible for controlling chiasma frequency. In the major part of the arms of the $\mathrm{L}$ and $\mathrm{M}$ chromosomes there are no G-bands but there is still considerable variation in chiasma frequency (fig. 2). Rather, the G-bands seem to represent a gross mechanism which suppresses or even eliminates chiasma formation within its confines and appears to be superimposed upon finer mechanisms controlling chiasma frequency variation along the chromosomes.

Acknowledgment.-We would like to thank the Centre for Overseas Pest Research who supplied some of the locusts used in these investigations and Professor F. W. Robertson for his comments on the manuscript.

$31 / 2-\mathrm{S} 2$ 


\title{
4. REFERENGES
}

CRAIG-CAMERon, T. A. 1971. Asynchronous chromosome replication at the end of the premeiotic DNA synthesis in Schistocerca gregaria (Forskảl). Cytobios, 4, 7-19.

FoX, D. P. 1966. The effects of X-rays on the chromosomes of locust embryos II. Chromatid interchanges and the organisation of the interphase nucleus. Chromosoma (Berl.), 20, 173-194.

Fox, D. P. 1973. The control of chiasma distribution in the locust, Schistocerca gregaria (Forskál). Chromosoma (Berl.), in press.

JOHN, B., AND LEWIS, K. R. 1965. The meiotic system. Protoplasmatologia, VI, F1, 1-335. Springer-Verlag, Wien.

JOHN, B., AND NAYLOR, B. 1961. Anomalous chromosome behaviour in the germ line of Schistocerca gregaria. Heredity, 16, 187-198.

LEE, C. L. Y., WELCH, J. P., AND LEE, S. H. s. 1973. Banding of human chromosomes by protein denaturation. Nature New Biology, 241, 142-143.

PARIS CONFERENCE (1971). 1972. Standardisation in human cytogenetics. Cytogenetics, 11, 313-362.

SAVAGE, J. R. K. 1973. The participation of human chromosome arms in radiation-induced chromatid exchange. Chromosomes Today, IV, in press.

BEABRIGHT, $M$. 1971. A rapid banding technique for human chromosomes. Lancet, $i i, 971$. SEABRIGHT, M. 1973. High resolution studies on the pattern of induced exchanges in the human karyotype. Chromosoma (Berl.), 40, 333-346.

SHIRAISHI, Y., AND YOSIDA, T. H. 1972. Banding pattern analysis of human chromosomes by use of a urea treatment technique. Chromosoma (Berl.), 37, 75-83.

SUMNER, A. T., EVANS, H. J., AND BUCKLAND, R. A. 1971. New technique for distinguishing between human chromosomes. Nature New Biology, 232, 31-32.

WANG, H. C., AND FEDOROFP, s. 1972. Banding pattern with Giemsa staining after treatment with ttypsin. Nature New Biology, 235, 52-53.

\section{FIELD OBSERVATIONS ON THE CYANOGENESIS POLYMORPHISM IN TRIFOLIUM REPENS}

\author{
J. P. A. ANGSEESING and W. J. ANGSEESING \\ St Poul's College, Cheltenhom GL5O 4AZ
}

Received 30.iv.73

\section{SUMmary}

We surveyed a population of Trifolium repens $\mathrm{L}$. polymorphic for cyanogenesis to determine whether either of two biotic factors were concerned as selective forces affecting the balance between the cyanogenic and acyanogenic forms.

We found no difference between the distributions of cyanogenesis scores for uninfected leaves and for leaves infected by the fungus Cymadothea trifolii Wolf. Acyanogenic plants, however, were more heavily eaten than cyanogenic ones, the principle herbivores being the slugs Arion ater L. and Agriolimax reticulatus Muller. Selective cating of isolated plants in a herbaceous border was due both to more acyanogenic leaves being touched and to more being eaten per a cyanogenic leaf. For plants embedded in the lawn selective eating was due mainly to the latter cause.

Possible explanation for the differences in degree of selective eating between lawn plants and border plants and between this survey and choice experiments are discussed.

\section{INTRODUCTION}

Most populations of Trifolium repens L. in Europe are polymorphic for cyanogenesis, with the leaves of cyanogenic plants releasing $\mathrm{HCN}$ gas when they 